\title{
Analysis of HAM-D scores and working ability in an observational study of Japanese patients with major depressive disorder and painful physical symptoms treated with duloxetine or SSRI monotherapy
}

This article was published in the following Dove Medical Press journal: Neuropsychiatric Disease and Treatment

\section{Atsushi Kuga ${ }^{1, *}$ \\ Tempei Otsubo ${ }^{2, *}$ \\ Toshinaga Tsuji ${ }^{3}$ \\ Shinji Hayashi ${ }^{3}$ \\ Hideyuki Imagawa' \\ Shinji Fujikoshi' \\ Rodrigo Escobar ${ }^{4}$}

'Medicines Development Unit Japan, Eli Lilly Japan K.K., Kobe, Japan;

${ }^{2}$ Department of Psychiatry, Tokyo Women's Medical University, Medical Center East, Tokyo, Japan; ${ }^{3}$ Medical Affairs Department, Shionogi \& Co., Ltd., Osaka, Japan; ${ }^{4}$ Bio-Medicines Global Team, Eli Lilly and Company, Madrid, Spain

*These authors contributed equally to this work
Correspondence: Atsushi Kuga Medicines Development Unit Japan, Eli Lilly Japan K.K., Lilly Plaza One Bldg. 5-I-28, Isogamidori, Chuo-ku, Kobe, Hyogo 65I-0086, Japan

$\mathrm{Tel}+8 \mathrm{I} 782424579$

Fax +8I 782429939

Email kuga_atsushi@lilly.com
Objective: To investigate the relationship between Hamilton Depression Rating Scale (HAM-D) score and psychiatrists' judgment of working ability in patients with major depressive disorder (MDD) and painful physical symptoms.

Methods: This was a prospective, observational, 12-week study in patients who received duloxetine or a selective serotonin reuptake inhibitor. Patients were $\geq 20$ years old, resided in Japan, and had at least moderate depression (Quick Inventory of Depressive Symptomatology $\geq 16$ ) and at least moderate painful physical symptoms (Brief Pain Inventory-Short Form average pain $\geq 3$ ). The main outcome in this post-hoc analysis was the HAM-D17 cutoff best corresponding with patients' working ability according to the investigator's judgment. Area under the receiver-operator curve was used to determine the time point with the strongest relationship between HAM-D17 and working ability. The optimal HAM-D17 cutoff was determined based on the maximum of sensitivity (true positive rate) minus ([1 minus specificity] [true negative rate]). For the evaluation of binary data, a mixed effects model with repeated measures analysis was used.

Results: For the estimation of the HAM-D17 cutoff, the area under the receiver-operator curve was maximal at 12 weeks, when a HAM-D17 score of 6 resulted in the best correspondence with working ability in the combined study population. At 12 weeks, a HAM-D17 score of 6 also resulted in the maximum predictive ability in each of the two treatment groups separately. For predicted working ability at 12 weeks, $52.7 \%$ of duloxetine-treated patients achieved the HAM-D17 cutoff of $\leq 6$, whereas $48.5 \%$ of SSRIs-treated patients achieved HAM-D17 $\leq 6$ $(P=0.477)$.

Conclusion: In this study of patients with major depressive disorder and painful physical symptoms, a HAM-D17 score $\leq 6$ corresponded best with patients' working ability. This finding is consistent with previous studies showing that a HAM-D17 cutoff of $\leq 7$ may overestimate functional recovery from MDD.

Keywords: functional recovery, reinstatement, remission

\section{Plain language summary}

The Hamilton Depression Rating Scale (HAM-D) is often used to assess depressive symptoms in clinical studies. While a HAM-D 17 item (HAM-D17) cutoff of $\leq 7$ has generally been used to define remission, many patients below that cutoff do not consider themselves ready 
to return to work. In the present analysis, we assessed the relationship between HAM-D17 scores and psychiatrists' assessments of patients' working ability. This analysis was performed on data from a 12-week observational study of duloxetine or a selective serotonin reuptake inhibitor in patients with both major depressive disorder (MDD) and painful physical symptoms (PPS). Based on the data at 12 weeks, when the largest HAM-D17 improvement was seen, we found that a HAM-D17 cutoff of $\leq 6$ had the best correspondence with patients' working ability. This was true in the combined treatment groups, and also when assessed for each treatment group separately. These findings are consistent with previous studies showing that a HAM-D17 cutoff of $\leq 7$ may be too high in terms of corresponding best with functional recovery from MDD.

\section{Introduction}

MDD is a chronic, prevalent, psychiatric disease with a substantial disease burden on health, social, and economic status. ${ }^{1-3}$ The loss of productivity due to absenteeism (prolonged absence from work) and presenteeism (working despite medical illness) is profound, and therapeutic approaches toward reinstatement (return to work) have been investigated. ${ }^{4,5}$ However, patients with MDD often relapse, ${ }^{2}$ making it difficult to return to work. While remission is the best indicator that patients may return to work, currently no biomarkers have been validated for the diagnosis of MDD and/or its remission. ${ }^{6}$ Given the lack of available biomarkers, numerous subjective measurement scales have been developed to assess various aspects of MDD. ${ }^{7}$ Some clinical scales, such as the Hamilton Depression Rating Scale ${ }^{8}$ and Montgomery-Asberg Depression Rating Scale, ${ }^{9}$ which count the number of existing symptoms and their severity, have been commonly used across many studies and clinical trials of antidepressants for patients with MDD.

Based on such established scales and the conceptualization of disease status, symptomatic resolution was believed to be close to remission, which is generally defined by a HAM-D17 score $\leq 7 .{ }^{10}$ Early clinical trials for patients with depression reported that remission would be followed by functional improvement, which is a critical component of recovery; ${ }^{11,12}$ however, some studies have described discordant results between functional recovery and remission of depressive symptoms. ${ }^{13-17}$ For example, according to a study by Zimmerman et al, ${ }^{16}$ about half of remitted patients evaluated based on a HAM-D17 score $\leq 7$ did not consider themselves to be remitted. With regard to reinstatement, patients who considered themselves to be in remission reported significantly better work performance than patients who did not consider themselves in remission. ${ }^{16}$ These results suggest that remission defined by a HAM-D17 score $\leq 7$ may not represent a sufficiently stringent criterion for reinstatement.

In addition to depressive symptoms per se, PPS are often observed in MDD, and their presence is associated with more severe depression. ${ }^{18}$ At least moderately PPS are also more common in Japanese patients with partial remission than in those with complete remission (based on a HAM-D17 score of $\leq 7) .{ }^{19}$ In a recent study of Japanese patients, the presence of PPS after 12 weeks of antidepressant treatment was significantly associated with failure to recover complete social and occupational functioning after 12 additional weeks of treatment, even among patients who had complete remission of MDD based on a HAM-D17 score of $\leq 7 .^{20}$

The present analysis assessed data from a 12-week, prospective, observational trial of patients with MDD and PPS to investigate improvement of PPS, depression, function, and quality-of-life between groups treated with duloxetine or selective serotonin reuptake inhibitors (SSRIs). ${ }^{21,22}$ In addition to the primary study outcomes, data were also collected on patients' ability to work based on investigators' judgment. In the present post-hoc analysis, we investigated the relationship between the psychiatrists' objective judgment for working ability and HAM-D scores by attempting to identify the cutoff value of the HAM-D17 score corresponding to working ability.

\section{Methods \\ Study design}

This was a prospective, observational, 12-week study in patients with MDD and PPS who received monotherapy with duloxetine $(n=273)$ or an SSRI $(n=250$; SSRIs were escitalopram, sertraline, paroxetine, or fluvoxamine). Full study methods and results of the primary efficacy outcomes and subgroup analyses have been reported previously. ${ }^{21,22}$ In brief, patients were $\geq 20$ years old, resided in Japan, and presented with an episode of MDD without psychotic traits as defined by the Diagnostic and Statistical Manual of Mental Disorders, 4th Edition, Text Revision. ${ }^{23}$ Patients had been diagnosed with at least moderate levels of both depression ( $\geq 16$, Quick Inventory of Depressive Symptomatology) ${ }^{24}$ and PPS (average pain $\geq 3$, Brief Pain Inventory-Short Form). ${ }^{25}$ Patients were excluded from participating in the study for any of the following: Prior diagnosis of schizophrenia, bipolar disorder, or other psychotic disorder; current diagnosis of 
dysthymic disorder or adjustment disorder; PPS that originated from organic disease aside from MDD; or treatment with opioids for PPS.

The study was conducted at 39 psychiatry and psychosomatic outpatient/inpatient clinic/hospital sites. The first and last patient visits were on February 13, 2014 and February 26, 2016, respectively.

\section{Measures}

Outcome measures for the current analysis included the HAM-D17, rated according to the Structured Interview Guide for the HAM-D17 for depressive symptoms, and working ability, as defined by the patient being able to work according to the investigators' judgment (yes/no). Both the HAM-D17 and working ability were assessed at weeks 4, 8, and 12 .

\section{Statistical methods}

The relationship between HAM-D17 and working ability was assessed for each corresponding visit evaluation (eg, Week 4 HAM-D17 and Week 4 working ability). Receiver-operating characteristic curves (ROCs) plotted sensitivity versus specificity. Sensitivity was the proportion of patients who achieved the given HAM-D17 score or less in patients with judgment of the ability to work of "Yes" (positive outcome). Specificity was the proportion of patients who did not achieve the given HAM-D17 score in patients with judgment of the ability to work of "No". The optimal cutoff was determined based on the maximum value of sensitivity (true positive rate) minus ([1 minus specificity] [true negative rate]), which provides equal weighting to detecting true positives and true negatives. Binary data were analyzed using a mixed effects model with repeated measures. The fixed effects model included treatment (duloxetine or SSRI), propensity score, baseline score visit, the visit-by-treatment interaction, the visit-by-propensity score interaction, and the visit-by-baseline score interaction. Statistical analyses were performed using SAS version 9.13 or above (SAS Institute Inc., Cary, NC, USA).

\section{Results}

First, in order to estimate the cutoff for working ability, we examined the relationship between the patients' working ability and HAM-D17 score at 4, 8, and 12 weeks of posttreatment for the combined treatment population. As shown in the ROCs in Figure 1, the area under the curve (AUC) increased, starting at 4 weeks, and was maximal at 12 weeks. We therefore estimated the cutoff based on data from the 12 -week visit. At 12 weeks, the cutoff of a HAM-D17 score of 6 resulted in the maximum value for sensitivity minus (1 minus specificity) of 0.602 (Table 1 ).

As a sensitivity analysis of the HAM-D17 cutoff estimated for the combined treatment groups, we next examined the ROC curves in each of the treatment groups separately at 12 weeks (Figure 2; Table S1). Consistent with the results shown in Figure 1, at 12 weeks a HAM-D17 score of 6 resulted in the maximum score of sensitivity minus ( 1 minus specificity) in each of the two treatment groups separately.

Given that the result for the combined population was confirmed for each treatment arm separately, we estimated that a HAM-D17 score $\leq 6$ had the best predictive value for patients' working ability. We therefore estimated the percentage of patients who reached HAM-D17 $\leq 6$ in the duloxetine and SSRI treatment groups at each time point (Figure 3 ). At 12 weeks, $52.7 \%$ of duloxetine-treated patients achieved HAM-D17 $\leq 6$, whereas $48.5 \%$ of SSRI-treated patients achieved HAM-D17 $\leq 6$; the difference in the proportion of
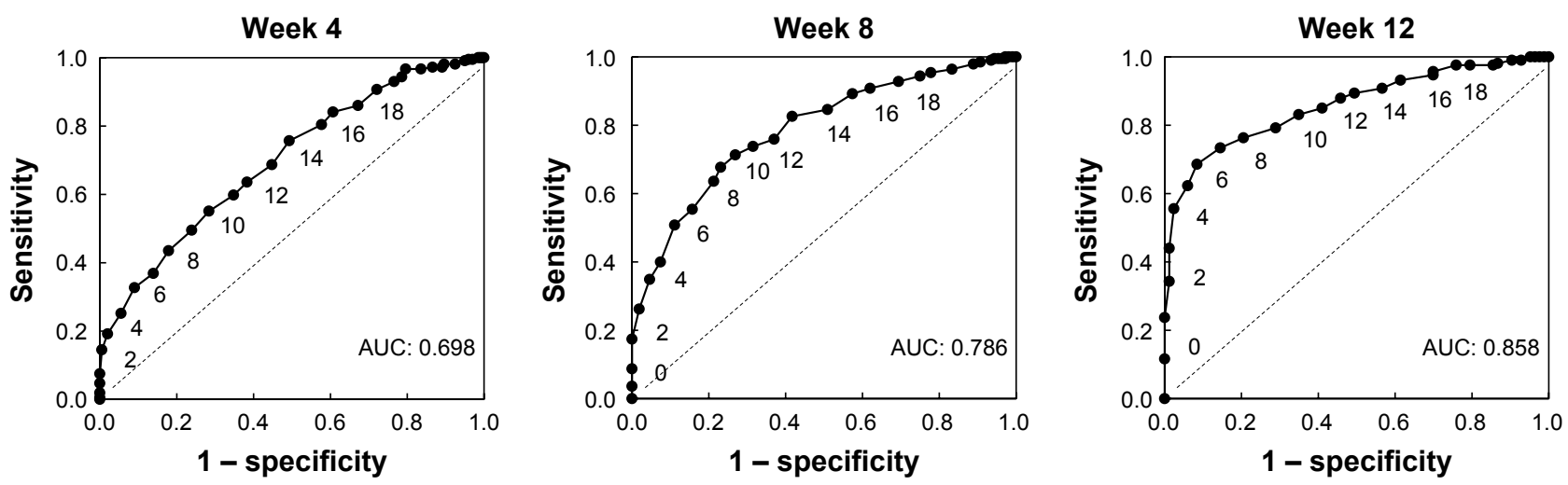

Figure I Receiver-operator curves at 4, 8, and 12 weeks, plotting each HAM-DI7 score in the total patient population. Data labels indicate HAM-DI7 scores. Abbreviations: AUC, area under the curve; HAM-D, Hamilton Depression Rating Scale. 


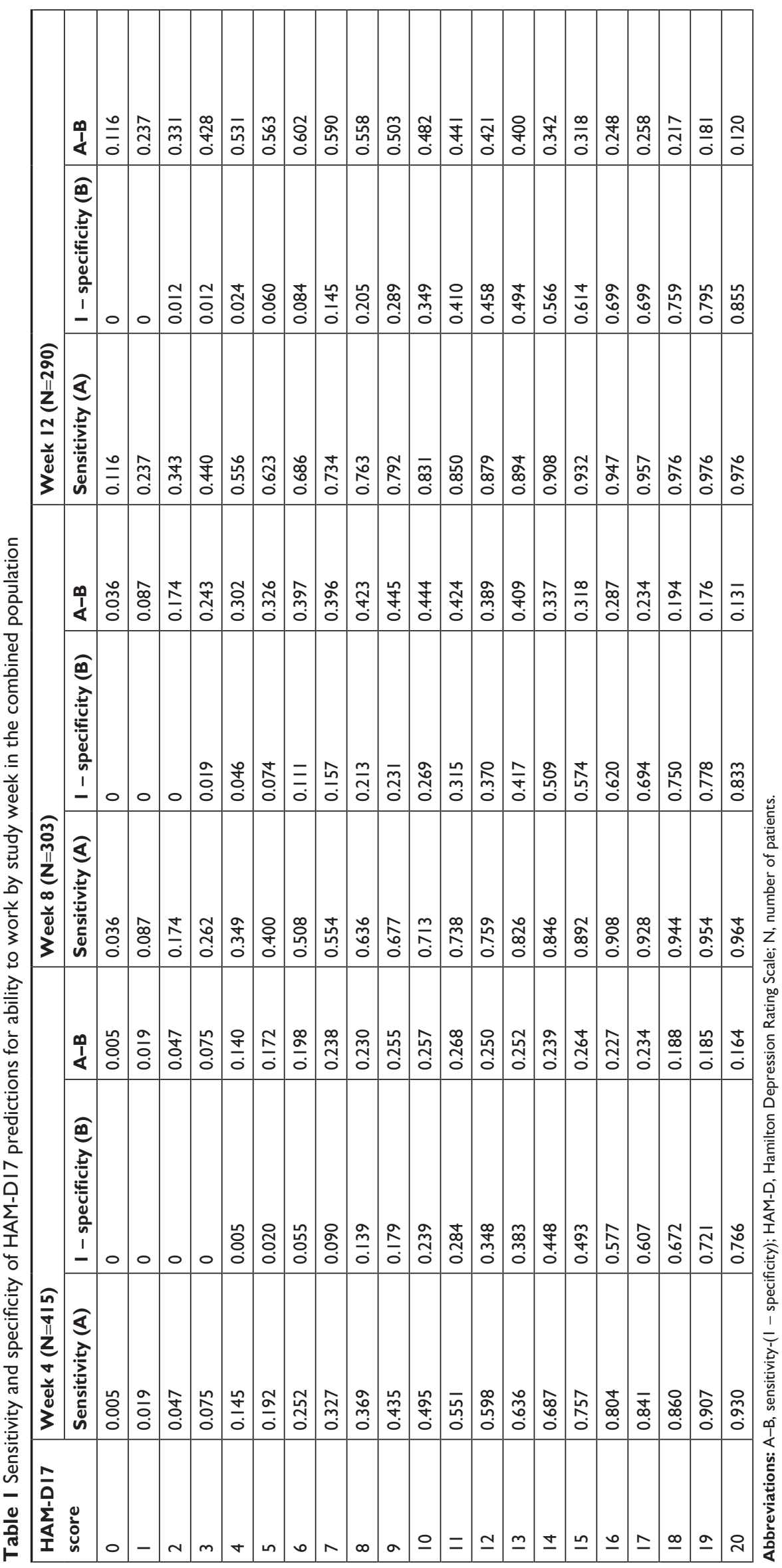


Duloxetine (Week 12)

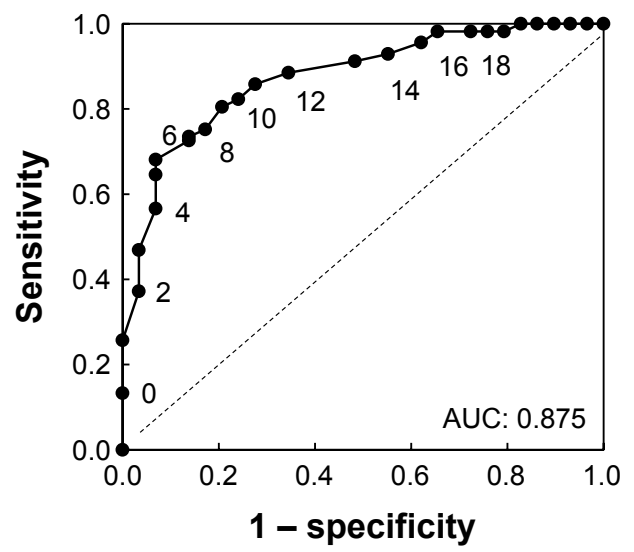

SSRIs (Week 12)

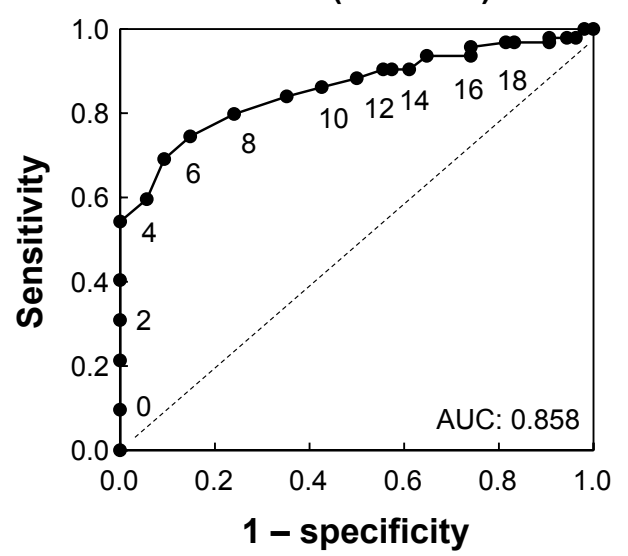

Figure 2 Receiver-operator curves plotting each HAM-DI7 score at 12 weeks for duloxetine- or SSRI-treated patients. Data labels indicate HAM-DI7 scores. Abbreviations: AUC, area under the curve; HAM-D, Hamilton Depression Rating Scale; SSRIs, selective serotonin reuptake inhibitors.

patients responding was not significantly different between treatment groups $(P=0.477)$.

\section{Discussion}

In the present analysis, we assessed the relationship between HAM-D17 scores and working ability in an observational clinical trial comparing duloxetine versus SSRI monotherapy in Japanese patients with MDD and PPS. At 12 weeks, in both the combined treatment group and in each treatment group separately, a HAM-D17 score of $\leq 6$ corresponded most closely with investigators' judgment of patients' working ability at the same time point.

A HAM-D17 score of 7 has historically been considered the cutoff value for remission; ${ }^{10}$ however, recently the idea that symptomatic improvement does not fully represent functional recovery has caused this notion to be revisited. ${ }^{13-17}$

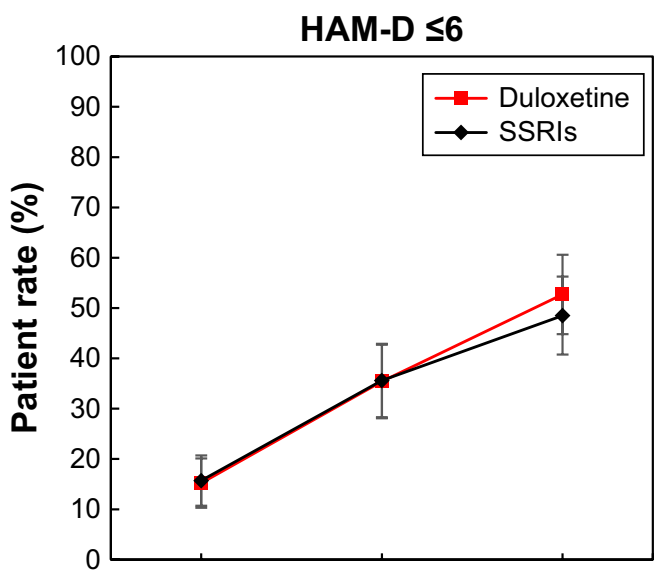

\begin{tabular}{lccc}
\hline Visit weeks & $\mathbf{4}$ & $\mathbf{8}$ & $\mathbf{1 2}$ \\
\hline $\begin{array}{l}\text { Duloxetine } \\
\mathrm{N}\end{array}$ & 210 & 153 & 147 \\
Rate & 15.2 & 35.4 & 52.7 \\
$(95 \% \mathrm{Cl})$ & $(10.9,20.7)$ & $(28.4,43.0)$ & $(44.7,60.5)$ \\
\hline SSRIs & & & \\
$\mathrm{N}$ & 212 & 160 & 152 \\
Rate & 15.7 & 35.6 & 48.5 \\
$(95 \% \mathrm{Cl})$ & $(11.4,21.4)$ & $(28.6,43.2)$ & $(40.8,56.3)$ \\
\hline -value MMRM & 0.880 & 0.969 & 0.477 \\
\hline
\end{tabular}

Figure 3 Proportion of patients achieving HAM-DI7 $\leq 6$ for duloxetine- and SSRI-treated patients. Error bars indicate $95 \%$ Cls.

Abbreviations: HAM-D, Hamilton Depression Rating Scale; MMRM, mixed measures repeated modeling analysis; N, number of patients; SSRIs, selective serotonin reuptake inhibitors. 
Romera et $\mathrm{al}^{27}$ examined the relationship between symptomatic improvement, assessed using the HAM-D17, and working ability, as assessed by objective evaluation using the Social and Occupational Functioning Assessment Scale (SOFAS) score. That analysis determined that a HAM-D17 score $\leq 5$ maximized sensitivity and specificity for identifying normal social and occupational function (based on a SOFAS score $\geq 80$ ). In another study using data from a postmarketing study of paroxetine in Japanese patients, Sawamura et $\mathrm{al}^{28}$ examined the relationship between HAM-D17 scores and quality-of-life scores on the 36-item Short Form Health Survey (SF-36). In that study, a HAM-D17 score $\leq 4$ was the best candidate for indicating recovery of social function, and improvements in the physical function and bodily pain domains were also significantly associated with this cutoff value.

The present findings are important in that absenteeism and presenteeism due to MDD are common problems worldwide, and result in substantial social costs. ${ }^{4,5}$ In daily practice, psychiatrists must make judgments about whether patients responding to out-patient treatment for MDD have recovered sufficiently to return to work; however, a proportion of those patients will still have impaired occupational function, as other studies have reported. ${ }^{29,30}$ Although scoring systems to evaluate functioning such as SOFAS and the SF-36 are available and well-validated, they may be time-consuming and are not widely used in clinical practice. In the present study of patients with MDD and PPS, we demonstrated that the clinicians' objective judgment for working ability would be compatible with a HAM-D17 score $\leq 6$. This result is consistent with the findings noted above that many patients with a HAM-D17 score of $\leq 7$ have not achieved complete functional recovery, although those studies generally pointed to even lower HAM-D cutoffs as corresponding best with full recovery.

The presence of PPS in MDD is very common, ${ }^{18,31}$ but the assessment of treatment outcomes in clinical practice for patients with MDD and PPS has not been fully investigated, particularly regarding measures of social functioning. We have previously observed a close relationship between MDD, PPS, and social functioning, including working ability, in a prospective observational study for 24 weeks. ${ }^{19,20}$ Notably, MDD patients with complete remission (defined as HAM-D17 $\leq 7$ ) at 12 weeks were more likely to achieve recovery of social functioning (judged by SOFAS $\geq 80$ ) at 24 weeks compared to those with partial remission (defined as HAM-D17 between 8 and 18, inclusive), ${ }^{20}$ which clearly suggests that symptomatic improvement is related to functional recovery. Importantly, with respect to PPS, completely-remitted patients with PPS at 12 weeks were less likely to achieve a SOFAS $\geq 80$ score at 24 weeks compared to completely-remitted patients without PPS at 12 weeks. It is also important to note that the HAM-D17 scale does not directly address PPS, as the only question on the instrument that specifically assesses physical symptoms is question 13, which refers to "general somatic symptoms" and thus does not distinguish between PPS and other physical symptoms. Given the relationship among PPS, MDD, and social functioning, and the lack of a direct measure of PPS in the HAM-D17 instrument, it is notable that we showed a HAM-D17 cutoff corresponding to occupational recovery in MDD with PPS. In other words, the presence of PPS as a comorbid symptom in subjects of this study might play a role in making the HAM-D17 cutoff value lower than 7 .

It is possible that the recovery of social functioning in patients with MDD might occur over an extended period of time. In a previous study in which two measures of social functioning (Global Assessment Scale and Social Adjustment Scale-Self Report) were monitored over 2 years, ${ }^{32}$ the measures showed continued amelioration from baseline to remission, remission to recovery, and after sustained recovery. In the current study, at 12 weeks, of 141 patients with a HAM-D17 score $\geq 7,76$ patients $(53.9 \%)$ were judged as Ability to work=No; of 15 patients with a HAM-D17 score of 7 , five patients (33.3\%) were judged as Ability to work=No. We could not investigate the temporal relationship between remission and recovery as our results were limited to only up to 12 weeks; however, these data suggest that a full recovery of social functioning might take longer than 12 weeks.

Previous research has examined functional recovery following treatment for MDD in numerous studies, including some that assessed response to different classes of agents such as serotonin-norepinephrine reuptake inhibitors versus SSRIs. ${ }^{33}$ Although results comparing between treatments varied, in general, functional recovery was seen across agents. In a randomized study of patients with severe MDD, treatment with duloxetine 60-120 mg daily resulted in significantly greater improvement in the Sheehan Disability Scale (SDS) compared to treatment with generic SSRIs. ${ }^{34}$ In a study comparing duloxetine 80 or $120 \mathrm{mg}$ /day to paroxetine $20 \mathrm{mg}$ QD, both treatments were superior to placebo for improvements on the SDS; however, no specific information comparing the active treatments for SDS improvement was 
provided. ${ }^{35}$ Finally, a meta-analysis of the norepinephrine reuptake inhibitor reboxetine versus SSRIs showed no significant difference in functional improvements between the active treatment groups based on the Social Adaptation Self-evaluation Scale. ${ }^{36}$ In the present study, we compared the proportion of patients who achieved the cutoff of a HAM-D17 score of $\leq 6$ in patients treated with duloxetine or with SSRIs. As shown in Figure 3, improvements were seen in both groups, with no significant difference between treatment arms.

\section{Limitations}

Limitations of the current study included the following: this was a post-hoc analysis, and the ability to work was not a primary or secondary study endpoint. As such, the indicator of "ability to work" was based on investigators' judgment and we did not collect actual data regarding patients' reinstatement, nor was the assessment by clinicians of ability to work validated as being comparable among investigators. In addition, the HAM-D17 may have been administered by the same investigator who rated patients' working ability; therefore, HAM-D17 scores may have influenced the investigators' assessment of working ability. It should also be noted that this study enrolled patients with MDD and at least moderate PPS, and results of this study cannot necessarily be generalized to MDD patients without PPS. Finally, this was an observational study without treatment randomization.

\section{Conclusion}

In the present study of patients with both MDD and PPS, the balance of sensitivity versus specificity for investigators' judgment of the ability to work was optimal at a HAM-D17 score $\leq 6$. This finding is consistent with previous studies showing that a HAM-D17 cutoff of $\leq 7$ may not best represent functional recovery from MDD.

\section{Ethics approval and consent}

The study was conducted in accordance with good postmarketing study practices ${ }^{26}$ and applicable Japanese laws and regulations. The Japanese Ministry of Health, Labor and Welfare reviewed and approved the protocol. Patients provided written informed consent before enrollment.

\section{Acknowledgments}

Medical writing assistance was provided by Thomas Melby of Syneos Health, funded by Eli Lilly Japan K.K. This study was funded by Eli Lilly Japan K.K.

\section{Author contributions}

All authors contributed to data analysis, drafting and revising the article, gave final approval of the version to be published, and agree to be accountable for all aspects of the work.

\section{Disclosure}

AK, HI, and SF are full-time employees of Eli Lilly Japan K.K.; AK and SF are stockholders of Eli Lilly and Company; TT and SH are full-time employees and stockholders of Shionogi \& Co., Ltd. TT also owns stock in Takeda Pharmaceutical Company Limited; RE is a full-time employee and stakeholder of Eli Lilly and Company. TO has received speaker's honoraria from Eli Lilly Japan K.K., Otsuka Pharmaceutical Co., Ltd., and Pfizer Japan Inc. The authors report no other conflicts of interest in this work.

\section{References}

1. Kessler RC, Angermeyer M, Anthony JC, et al. Lifetime prevalence and age-of-onset distributions of mental disorders in the World Health Organization's World Mental Health Survey Initiative. World Psychiatry. 2007;6(3):168-176.

2. Mueller TI, Leon AC, Keller MB, et al. Recurrence after recovery from major depressive disorder during 15 years of observational follow-up. Am J Psychiatry. 1999;156(7):1000-1006. doi:10.1176/ajp. 156.7.1000

3. Bromet E, Andrade LH, Hwang I, et al. Cross-national epidemiology of DSM-IV major depressive episode. BMC Med. 2011;9:90. doi:10.1186/1741-7015-9-90

4. de Graaf R, Tuithof M, van Dorsselaer S, ten Have M. Comparing the effects on work performance of mental and physical disorders. Soc Psychiatry Psychiatr Epidemiol. 2012;47(11):1873-1883. doi:10.1007/ s00127-012-0496-7

5. Ekman M, Granström O, Omérov S, Jacob J, Landén M. The societal cost of depression: evidence from 10,000 Swedish patients in psychiatric care. J Affect Disord. 2013;150(3):790-797. doi:10.1016/j. jad.2013.03.003

6. Fonseka TM, MacQueen GM, Kennedy SH. Neuroimaging biomarkers as predictors of treatment outcome in major depressive disorder. $J$ Affect Disord. 2018;233:21-35. doi:10.1016/j.jad.2017.10.049

7. Möller HJ. Standardised rating scales in psychiatry: methodological basis, their possibilities and limitations and descriptions of important rating scales. World J Biol Psychiatry. 2009;10(1):6-26. doi:10.1080/15622970802264606

8. Hamilton M. A rating scale for depression. J Neurol Neurosurg Psychiatry. 1960;23:56-62.

9. Montgomery SA, Asberg M. A new depression scale designed to be sensitive to change. Br J Psychiatry. 1979;134:382-389.

10. Frank E, Prien RF, Jarrett RB, et al. Conceptualization and rationale for consensus definitions of terms in major depressive disorder. Remission, recovery, relapse, and recurrence. Arch Gen Psychiatry. 1991;48(9): 851-855.

11. Miller IW, Keitner GI, Schatzberg AF, et al. The treatment of chronic depression, part 3: psychosocial functioning before and after treatment with sertraline or imipramine. J Clin Psychiatry. 1998;59(11): 608-619.

12. Papakostas GI, Petersen T, Denninger JW, et al. Psychosocial functioning during the treatment of major depressive disorder with fluoxetine. J Clin Psychopharmacol. 2004;24(5):507-511. 
13. Hirschfeld RM, Dunner DL, Keitner G, et al. Does psychosocial functioning improve independent of depressive symptoms? A comparison of nefazodone, psychotherapy, and their combination. Biol Psychiatry. 2002;51(2):123-133.

14. van der Voort TY, Seldenrijk A, van Meijel B, et al. Functional versus syndromal recovery in patients with major depressive disorder and bipolar disorder. J Clin Psychiatry. 2015;76(6):e809-e814. doi:10.4088/ JCP. $14 \mathrm{~m} 09548$

15. Zimmerman M, McGlinchey JB, Posternak MA, Friedman M, Boerescu D, Attiullah N. Discordance between self-reported symptom severity and psychosocial functioning ratings in depressed outpatients: implications for how remission from depression should be defined. Psychiatry Res. 2006;141(2):185-191. doi:10.1016/j. psychres.2005.05.016

16. Zimmerman M, Martinez JA, Attiullah N, et al. Why do some depressed outpatients who are in remission according to the Hamilton Depression Rating Scale not consider themselves to be in remission? J Clin Psychiatry. 2012;73(6):790-795. doi:10.4088/JCP.11m07203.

17. Bortolato B, Miskowiak KW, Köhler CA, et al. Cognitive remission: a novel objective for the treatment of major depression? BMC Med. 2016;14:9. doi:10.1186/s12916-016-0560-3

18. Brnabic A, Lin C, Monkul ES, Dueñas H, Raskin J. Major depressive disorder severity and the frequency of painful physical symptoms: a pooled analysis of observational studies. Curr Med Res Opin. 2012;28(12):1891-1897. doi:10.1185/03007995.2012.748654

19. Harada E, Satoi Y, Kikuchi T, Watanabe K, Alev L, Mimura M. Residual symptoms in patients with partial versus complete remission of a major depressive disorder episode: patterns of painful physical symptoms in depression. Neuropsychiatr Dis Treat. 2016;12:1599-1607.

20. Harada E, Satoi Y, Kuga A, et al. Associations among depression severity, painful physical symptoms, and social and occupational functioning impairment in patients with major depressive disorder: a 3-month, prospective, observational study. Neuropsychiatr Dis Treat. 2017;13:2437-2445. doi:10.2147/NDT.S134566

21. Kuga A, Tsuji T, Hayashi S, et al. An observational study of duloxetine versus SSRI monotherapy for the treatment of painful physical symptoms in Japanese patients with major depressive disorder: primary analysis. Neuropsychiatr Dis Treat. 2017;13:2105-2114. doi:10.2147/ NDT.S131438

22. Kuga A, Tsuji T, Hayashi S, et al. An observational study of duloxetine versus SSRI monotherapy in Japanese patients with major depressive disorder: subgroup analyses of treatment effectiveness for pain, depressive symptoms, and quality of life. Neuropsychiatr Dis Treat. 2017;13:2115-2124. doi:10.2147/NDT.S136448

23. Diagnostic and Statistical Manual of Mental Disorders. 4th ed. Text Revison. Washington (DC): American Psychiatric Association; 2000.

24. Rush AJ, Trivedi MH, Ibrahim HM, et al. The 16-Item Quick Inventory of Depressive Symptomatology (QIDS), clinician rating (QIDS-C), and self-report (QIDS-SR): a psychometric evaluation in patients with chronic major depression. Biol Psychiatry. 2003;54(5):573-583.
25. Cleeland CS, Ryan KM. Pain assessment: global use of the brief pain inventory. Ann Acad Med Singapore. 1994;23(2):129-138.

26. Ministry of Health, Labor, and Welfare. Good Post-Marketing Study Practices. Ordinance No. 171 issued on December 20; 2004.

27. Romera I, Pérez V, Menchón JM, Polavieja P, Gilaberte I. Optimal cutoff point of the Hamilton Rating Scale for Depression according to normal levels of social and occupational functioning. Psychiatry Res. 2011;186(1):133-137. doi:10.1016/j.psychres.2010.06.023

28. Sawamura J, Ishigooka J, Nishimura K. Re-evaluation of the definition of remission on the 17-item Hamilton Depression Rating Scale based on recovery in health-related quality of life in an observational post-marketing study. Health Qual Life Outcomes. 2018;16(1):14. doi:10.1186/s12955-018-0838-6

29. de Vries G, Koeter MW, Nieuwenhuijsen K, Hees HL, Schene AH. Predictors of impaired work functioning in employees with major depression in remission. J Affect Disord. 2015;185:180-187. doi:10.1016/j. jad.2015.07.013

30. Trivedi MH, Morris DW, Wisniewski SR, et al. Increase in work productivity of depressed individuals with improvement in depressive symptom severity. Am J Psychiatry. 2013;170(6):633-641. doi:10.1176/ appi.ajp.2012.12020250

31. Kishi T, Matsuda Y, Mukai T, et al. A cross-sectional survey to investigate the prevalence of pain in Japanese patients with major depressive disorder and schizophrenia. Compr Psychiatry. 2015;59:91-97. doi:10.1016/j.comppsych.2015.02.004

32. Furukawa TA, Takeuchi H, Hiroe T, et al. Symptomatic recovery and social functioning in major depression. Acta Psychiatr Scand. 2001; 103(4):257-261.

33. Sheehan DV, Nakagome K, Asami Y, Pappadopulos EA, Boucher M. Restoring function in major depressive disorder: a systematic review. J Affect Disord. 2017;215:299-313. doi:10.1016/j.jad.2017.02.029.

34. Martinez JM, Katon W, Greist JH, et al. A pragmatic 12-week, randomized trial of duloxetine versus generic selective serotonin-reuptake inhibitors in the treatment of adult outpatients in a moderate-to-severe depressive episode. Int Clin Psychopharmacol. 2012;27(1):17-26. doi:10.1097/YIC.0b013e32834ce11b

35. Detke MJ, Wiltse CG, Mallinckrodt CH, McNamara RK, Demitrack MA, Bitter I. Duloxetine in the acute and long-term treatment of major depressive disorder: a placebo- and paroxetine-controlled trial. Eur Neuropsychopharmacol. 2004;14(6):457-470. doi:10.1016/j.euroneuro. 2004.01.002

36. Papakostas GI, Nelson JC, Kasper S, Möller HJ. A meta-analysis of clinical trials comparing reboxetine, a norepinephrine reuptake inhibitor, with selective serotonin reuptake inhibitors for the treatment of major depressive disorder. Eur Neuropsychopharmacol. 2008;18(2):122-127. doi:10.1016/j.euroneuro.2007.07.005 


\section{Supplementary material}

Table SI Sensitivity, specificity, and AUC of HAM-DI7 predictions for ability to work at Week 12 for duloxetine and SSRIs

\begin{tabular}{|c|c|c|c|c|c|c|}
\hline \multirow[t]{2}{*}{ HAM-DI 7 score } & \multicolumn{3}{|c|}{$\begin{array}{l}\text { Duloxetine } \\
(\mathrm{N}=142)(\mathrm{AUC}=\mathbf{0 . 8 7 5})\end{array}$} & \multicolumn{3}{|c|}{$\begin{array}{l}\text { SSRIs } \\
(N=\mid 48)(A \cup C=0.858)\end{array}$} \\
\hline & Sensitivity (A) & I - specificity (B) & A-B & Sensitivity (A) & I - specificity (B) & A-B \\
\hline 0 & 0.133 & 0 & 0.133 & 0.096 & 0 & 0.096 \\
\hline I & 0.257 & 0 & 0.257 & 0.213 & 0 & 0.213 \\
\hline 2 & 0.372 & 0.034 & 0.337 & 0.309 & 0 & 0.309 \\
\hline 3 & 0.469 & 0.034 & 0.435 & 0.404 & 0 & 0.404 \\
\hline 4 & 0.566 & 0.069 & 0.497 & 0.543 & 0 & 0.543 \\
\hline 5 & 0.646 & 0.069 & 0.577 & 0.596 & 0.056 & 0.540 \\
\hline 6 & 0.681 & 0.069 & 0.612 & 0.691 & 0.093 & 0.599 \\
\hline 7 & 0.726 & 0.138 & 0.588 & 0.745 & 0.148 & 0.597 \\
\hline 8 & 0.735 & 0.138 & 0.597 & 0.798 & $0.24 I$ & 0.557 \\
\hline 9 & 0.752 & 0.172 & 0.580 & 0.840 & 0.352 & 0.489 \\
\hline 10 & 0.805 & 0.207 & 0.598 & 0.862 & 0.426 & 0.436 \\
\hline II & 0.823 & $0.24 I$ & 0.582 & 0.883 & 0.500 & 0.383 \\
\hline 12 & 0.858 & 0.276 & 0.583 & 0.904 & 0.556 & 0.349 \\
\hline 13 & 0.885 & 0.345 & 0.540 & 0.904 & 0.574 & 0.330 \\
\hline 14 & 0.912 & 0.483 & 0.429 & 0.904 & 0.611 & 0.293 \\
\hline 15 & 0.929 & 0.552 & 0.377 & 0.936 & 0.648 & 0.288 \\
\hline 16 & 0.956 & 0.621 & 0.335 & 0.936 & $0.74 I$ & 0.195 \\
\hline 17 & 0.956 & 0.621 & 0.335 & 0.957 & $0.74 I$ & 0.217 \\
\hline 18 & 0.982 & 0.655 & 0.327 & 0.968 & 0.815 & 0.153 \\
\hline 19 & 0.982 & 0.724 & 0.258 & 0.968 & 0.833 & 0.135 \\
\hline 20 & 0.982 & 0.759 & 0.224 & 0.968 & 0.907 & 0.061 \\
\hline
\end{tabular}

Abbreviations: A-B, sensitivity-(I-specificity); AUC, area under the curve; HAM-D, Hamilton Depression Rating Scale; N, number of patients; SSRIs, selective serotonin reuptake inhibitors.

\section{Publish your work in this journal}

Neuropsychiatric Disease and Treatment is an international, peerreviewed journal of clinical therapeutics and pharmacology focusing on concise rapid reporting of clinical or pre-clinical studies on a range of neuropsychiatric and neurological disorders. This journa is indexed on PubMed Central, the 'PsycINFO' database and CAS, and is the official journal of The International Neuropsychiatric Association (INA). The manuscript management system is completely online and includes a very quick and fair peer-review system, which is all easy to use. Visit http://www.dovepress.com/testimonials.php to read real quotes from published authors. 